\title{
Kidney transplant Medicare payments and length of stay: associations with comorbidities and organ quality
}

Gerardo Machnicki¹, Krista L. Lentine ${ }^{1}$, Paolo R. Salvalaggio², Thomas E. Burroughs ${ }^{1}$, Daniel C. Brennan ${ }^{3}$, Mark A. Schnitzler ${ }^{1}$

${ }^{1}$ Center for Outcomes Research, Saint Louis University School of Medicine, Saint Louis, MO, USA

2Department of Surgery, University of Washington, Seattle, WA, USA

${ }^{3}$ Department of Medicine, Washington University, Saint Louis, MO, USA

Submitted: 8 June 2010

Accepted: 6 September 2010

Arch Med Sci 2011; 7, 2: 278-286

DOI: 10.5114/aoms.2011.22079

Copyright @ 2011 Termedia \& Banach

\section{Abstract}

Introduction: We investigated associations between pre-transplant comorbidities, length of stay (LOS) and Medicare payments for transplant hospitalization.

Material and methods: We examined United States Renal Data System for 24,963 recipients of first deceased-donor kidney transplants in 1995-2002 for whom Medicare was the primary payer for at least a year pre-transplant. Pre-transplant ICD-9-CM codes from claims were classified with the Charlson and Elixhauser algorithms. Regression models for payments and LOS included: 1) baseline recipient, donor and transplant factors from the Organ Procurement and Transplant Network (OPTN), 2) OPTN variables and individual comorbidities and 3) OPTN variables and counts of Charlson or Elixhauser comorbidities.

Results: Factors most strongly associated with LOS were type I diabetes, cold ischemia time $>36 \mathrm{~h}$, expanded criteria donor (ECD) and donation after cardiac death (DCD). Except for ECD, each was associated with increased payments. Upper respiratory disease, liver disease, peptic ulcer disease, diabetes, cancer and other diseases were also associated with increased LOS and payments. Each additional Charlson comorbidity increased LOS by $2.94 \%$ and payments by $\$ 471$ (Elixhauser results: $1.71 \%$ for LOS, \$277 for payments). Use of ECD or DCD organs were associated with $10-15 \%$ higher LOS and 5\% increased Medicare payments for DCD.

Conclusions: This methodology could be used to explore if Medicare reimbursement for transplantation of higher-risk recipients and using nonstandard organs is financially adequate and to analyze related questions in other healthcare systems.

Key words: kidney transplantation, comorbidities, lenght of stay cost.

\section{Introduction}

The analysis of the determinants of healthcare cost and reimbursement is a recurring topic in the transplant literature given the importance of adequate financial compensation to ensure the operation of transplant programs and support at a national level. Reimbursement for inpatient services by public and private payors uses a prospective approach that pays hospitals according to the type of procedure or diagnosis. In the United States (US), Diagnosis Related Groups (DRGs) were introduced by Medicare in 1983 to pay for hospital inpatient services, including kidney

\section{Corresponding author:}

Prof. Mark A. Schnitzler MD,

$\mathrm{PhD}$

Saint Louis University Center for Outcomes Research 3545 Lafayette Avenue Salus Center, $2^{\text {nd }}$ Floor Room 2825

St. Louis, MO 63104, USA

Phone: 314-977-9476

Fax: 314-977-1101

E-mail: schnitm@slu.edu 
transplantation. Diagnosis Related Groups-like systems are also used in many other countries. Diagnosis Related Groups separate patients into similar groups based on resource utilization to allow for a common reimbursement rate. While prospective payment systems are needed to control cost and foster efficiency, there are financial-risks for providers when a single DRG is used to reimburse for heterogeneous patient populations.

In the US, the transplant population is evolving towards a more severe case-mix, due for example to the use of more marginal organs or transplantation of patients with more comorbidities [1-4]. Diagnosis Related Groups adjustments compensate only for the most extreme outliers in transplantation, such as patients costing more than $\$ 45,000$ [5]. Twenty percent of transplant payments were in that category for Medicare between 2003 and 2006 [5]. However, a "gap" zone exists for more severe patients that do not reach this high cost [6] and concerns exist that current reimbursement may not be adequate in cases such as those receiving ECD or DCD kidneys [1-3]; while the same has been expressed for certain types of liver transplants [7].

Use of an expanded criteria donor (ECD) [1], donation after cardiac death donor (DCD) [2] and duration of dialysis longer than one year [8] have been associated with either inadequate payments or longer length of stay (LOS) for the transplant procedure admission. There are other factors that could influence transplant procedure costs. Comorbidities are one important factor. Comorbidities have been found to be associated with increased hospital costs across several conditions such as cardiovascular disease, respiratory conditions, asthma and others [9]. In transplantation, existing evidence supports that pre-transplant comorbidities such as history of cerebrovascular accident and chronic obstructive pulmonary disease (COPD) are associated with longer LOS for the transplant procedure [8]. The DRG for transplantation does not allow to adjust for comorbidities, complications or organ quality [5].

Most of the available evidence about comorbidities is derived from single center data. The advantage of some of such studies is availability of cost-information from hospital accounting systems; however the samples represent only a small percent of transplant centers. In addition, only a limited number of comorbidities have been studied.

The United States Renal Data System (USRDS) captures billing claims data for Medicare-insured transplant recipients across the country. Billing claims are diagnosis-linked and can provide additional evidence regarding the associations between pre-transplant comorbidites and financial outcomes in kidney transplantation. We examined USRDS data for Medicare-insured kidney transplant recipients to accomplish two major aims. First, we determined the associations between pretransplant comorbidities and transplant procedure LOS (considering LOS as a proxy for institutional costs). Second, we estimated the associations between Medicare payments for the transplant procedure and pre-transplant comorbidities in Medicare-insured patients. In both case we used an expanded set of comorbidities compared to the usual comorbidity information included in the Organ Procurement and Transplantation Network (OPTN) registry. Associations between donor organ type, LOS and payments were also estimated.

\section{Material and methods}

\section{Study population}

This retrospective cohort study included all adult (> 18 years) recipients of deceased-donor, first renal only transplants from 1995-2002 for whom Medicare was the primary insurer at the time of transplant. Patient information was obtained from the OPTN and included in the USRDS registry which incorporates transplant registry information from the OPTN.

\section{Comorbidity and payments and length of stay data from claims}

Comorbidity information from Medicare claims was identified using the Medicare Part-A institutional and Part -B physician/supplier claims files. Primary and secondary International Classification of Diseases, $9^{\text {th }}$ revision, clinical modification (ICD-9-CM) diagnosis codes for each hospitalization and physician visit during the last pre-transplant year were considered. In order to ascertain pretransplant comorbidities using an observation window of consistent duration across subjects, the sample was limited to patients with at least one continuous year of primary Medicare insurance before transplant. Transplant procedure Medicare payments and LOS were retrieved from the transplant procedure claims identified by DRG 302.

\section{Comorbidity summary measures}

The ICD-9 CM diagnostic codes were summarized using two comorbidity classification systems. The Charlson [10] and Elixhauser [11] algorithms classify diagnoses into 19 and 31 comorbidity groupings, respectively. Both are widely used measures of comorbidity in predictive models [12]. Adaptations of both indices for claims databases were used [13].

\section{Other study variables}

The OPTN variables describing recipient, donor and transplant factors were included in the 
regression models. These included recipient age, race and body mass index (BMI) (including missing $B M I)$, cause of ESRD, duration of pre-transplant dialysis, peak panel reactive antibodies (PRA) $\geq 50 \%$, OPTN-reported comorbidities; donor type (standard criteria deceased, ECD, and DCD), donor age (including missing age), race, BMI (including missing $B M I)$, and OPTN-reported comorbidties; number of donor-recipient ABDR HLA mismatches (none, $A B$ mismatches, DR mismatches), donor-recipient cytomegalovirus (CMV) sero-pairing, and categorized cold ischemia time. Delayed graft function (DGF) was excluded because it was considered a post-transplant outcomes variable. Immunosuppressant therapy at discharge and use of induction therapy were also excluded.

\section{Statistical analysis}

Associations between patient, donor and transplant characteristics with Medicare payments and LOS were estimated using multilevel regression models. Multilevel models allow estimating the effect of variables at different levels on a given outcome. In multilevel models, the parameters of one level can be modeled as outcomes following a distribution in another level [14]. This reduces the influence of the different variables to two types: fixed effects, for which coefficients and its variances are estimated and random effects that capture the variability at different levels (i.e. pertaining to the observations or to the modeled parameters). These models are adequate to account for clustering of observations (i.e. correlated errors) at different levels. In this case, it was hypothesized that clustering at the hospital level occurred, requiring a model that can account for the correlation among LOS and among payments at a given transplant center. Use of ordinary regression methods in the presence of correlated errors can produce low (biased) standard errors of the estimates and can show more statistically significant variables compared to the results of a multilevel model.

Multilevel linear regression models were built for Medicare payments as the dependent variable using OPTN variables and Charlson or Elixhauser comorbidities and OPTN variables and the sum of Charlson or Elixhauser comorbidities. Models with LOS as the dependent variable were built for each comorbidity classification system, containing the same dependent variables as the Medicare payment models. A generalized linear model with Poisson distribution and a log link was used for LOS, as it accounts for the discrete distribution of hospital days. Parameter estimates were expressed as percentage increase (or decrease) in LOS and as a dollar change in Medicare payment versus a reference. For dichotomous variables such as ECD or DCD, the reference case refers to patients without the variable of interest holding all other variables constant. When calculating Medicare payments and LOS in models with the sum of Charlson (range 0-6) or Elixhauser (range 0-11) comorbidities the results compare to patients without comorbidities while all other recipient, donor and transplant factors were held constant. In all models, the intercept was modeled as a random effect and therefore allowed differentiation by transplant center. All analyses were performed in SAS 9.1 (Cary, NC). Proc Mixed [15] was used for the Medicare payment models and Proc Glimmix [16] was used for the LOS models.

\section{Results}

\section{Patient population and comorbidities}

During this time period, 27,177 patients met initial study criteria: 3,167 patients $(12.7 \%)$ had missing donor age, 846 (3.39\%) had missing recipient $\mathrm{BMI}$ and 879 (3.52\%) lacked donor BMI. Thus, 24,963 patients remained after excluding patients with missing data in other variables. Most patients were between 30 and 59 years $(19,146$ [77.8\%]), 38,9\% were male and $35.11 \%$ were AfricanAmerican. The most prevalent OPTN reported comorbidities were hypertension ( $n=18,655$ [74.73\%]) and diabetes ( $n=7,607$ [30.47\%]). Twenty-five percent of patients had 3 or more Charlson comorbidities and twenty percent of patients had six or more Elixhauser comorbidities. Transplant induction therapy was used in 12,026 patients (48.18\%). The most prevalent immunosuppressive regimen at discharge was cyclosporine and mycophenolate mofetil ( $n=9,009,36.09 \%)$, with more than $90 \%$ of patients receiving steroids at discharge $(n=23,339,93.49 \%)$ (Table I).

\section{Regression estimates for transplant procedure and longer length of stay}

Organ Procurement and Transplantation Network factors

The LOS and payments for population average characteristics were 9.48 days and 27,594 respectively. Some of the top 10 OPTN variables associated with highest percentage increase in LOS and Medicare payments were common to both outcomes and included type I diabetes as cause of ESRD, cold ischemia time > $36 \mathrm{~h}$, DCD, dialysis duration longer than 5 years, the presence of DR mismatches, history of myocardial infarction and presence of functional limitations. Donor age (more than 44 years) and unreported CMV sero-pairing were also associated with longer LOS while Hispanic recipient and history of Chronic Obstructive Pulmonary Disease (COPD) were associated with higher Medicare payments (Table II). The results of 
Table I. Patient, donor and transplant characteristics of the study population

\begin{tabular}{|c|c|c|c|}
\hline Variable & $N(\%)(n=24,963)$ & Variable & $N(\%)(n=24,963)$ \\
\hline \multicolumn{2}{|l|}{ Recipient factors } & \multicolumn{2}{|l|}{ Donor factors } \\
\hline \multicolumn{2}{|l|}{ Age } & \multicolumn{2}{|l|}{ Age } \\
\hline $18-29$ & $2,220(8.89)$ & $<18$ & $3,795(15.2)$ \\
\hline $30-44$ & $7,395(29.62)$ & $18-29$ & $4,797(19.22)$ \\
\hline $45-59$ & $9,801(39.26)$ & $30-44$ & $5,354(21.45)$ \\
\hline $60+$ & $5,547(22.22)$ & $45-59$ & $5,808(23.27)$ \\
\hline Female gender & $9,703(38.87)$ & $60+$ & $2,042(8.18)$ \\
\hline \multicolumn{2}{|l|}{ Racial background } & Unknown & 3,167 (12.69) \\
\hline Black & $8,764(35.11)$ & Female gender & 9,968 (39.93) \\
\hline Other & $1,621(6.49)$ & \multicolumn{2}{|l|}{ Racial background } \\
\hline White & $14,578(58.4)$ & Black & $3,031(12.14)$ \\
\hline Hispanic origin & $3,248(13.01)$ & Other & $1,421(5.69)$ \\
\hline \multicolumn{2}{|l|}{ Body mass index } & White & $20,511(82.17)$ \\
\hline Normal & $10,699(42.86)$ & Hispanic origin & $2,709(10.85)$ \\
\hline Overweight & 7,895 (31.63) & \multicolumn{2}{|l|}{ Body mass index } \\
\hline Obese & $5,523(22.12)$ & Normal & $12,747(51.06)$ \\
\hline Unknown & $846(3.39)$ & Overweight & $6,506(26.06)$ \\
\hline Limits & $2,103(8.42)$ & Obese & $4,831(19.35)$ \\
\hline \multicolumn{2}{|l|}{ Primary cause of ESRD } & Unknown & $879(3.52)$ \\
\hline Type I diabetes & 2,938 (11.77) & \multicolumn{2}{|l|}{ Donor cause of death } \\
\hline Type II diabetes & $3,803(15.23)$ & Anoxia & 2,291 (9.18) \\
\hline Hypertension & $6,310(25.28)$ & Cerebrovascular/stroke & $9,789(39.21)$ \\
\hline Polycystic kidney disease (PKD) & $1,819(7.29)$ & Head trauma & $11,448(45.86)$ \\
\hline Glomeruloneprhitis & $4,751(19.03)$ & CNS tumor & $250(1)$ \\
\hline Other & $3,090(12.38)$ & Other & $1,185(4.75)$ \\
\hline Unknown & $2,252(9.02)$ & Donor comorbidities & \\
\hline \multicolumn{2}{|l|}{ Pre-tx dialysis duration } & Hypertension & $4,744(19.0)$ \\
\hline $12-24$ months & $4,472(17.91)$ & Diabetes & $855(3.43)$ \\
\hline $24-60$ months & $14,612(58.53)$ & Alcohol abuse & 4,539 (18.18) \\
\hline$>60$ months & $5,879(23.55)$ & Cigarette use & $9,344(37.43)$ \\
\hline \multicolumn{2}{|l|}{ OPTN recorded comorbidities } & Drug use & $4,382(17.55)$ \\
\hline Angina & $3,182(12.75)$ & \multicolumn{2}{|l|}{ Transplant characteristics } \\
\hline Arrhythmia & $184(0.74)$ & PRA $\geq 50 \%$ & $1,366(5.47)$ \\
\hline Congestive heart failure & $1,685(6.75)$ & \multicolumn{2}{|l|}{ HLA mismatches } \\
\hline COPD & $427(1.71)$ & No MM & $1,982(7.94)$ \\
\hline Cerebrovascular disease & $609(2.44)$ & No DR MM & $5,609(22.47)$ \\
\hline Hypertension & $18,655(74.73)$ & DR MM & $17,372(69.59)$ \\
\hline Myocardial infarction & $342(1.37)$ & \multicolumn{2}{|l|}{ Cold ischemia time } \\
\hline Diabetes & $7,607(30.47)$ & $0-12 \mathrm{~h}$ & $3,418(13.69)$ \\
\hline Peripheral vascular disease & $1,560(6.25)$ & $13-24 \mathrm{~h}$ & $11,991(48.04)$ \\
\hline Smoking history & $703(2.82)$ & $25-36 \mathrm{~h}$ & $6,288(25.19)$ \\
\hline \multirow[t]{2}{*}{ Alcohol abuse history } & $144(0.58)$ & $36+h$ & $1,014(4.06)$ \\
\hline & & Undetermined & $2,252(9.02)$ \\
\hline
\end{tabular}


Table I. cont.

\begin{tabular}{|c|c|c|c|}
\hline Variable & $N(\%)(n=24,963)$ & Variable & $N(\%)(n=24,963)$ \\
\hline \multicolumn{2}{|c|}{ Charlson comorbidities } & \multicolumn{2}{|l|}{ Cytomegalovirus sero-pairing } \\
\hline 0 & $6,302(25.25)$ & Donor-/recipient- & 2,892 (11.59) \\
\hline 1 & $7,446(29.83)$ & Donor-/recipient+ & $5,468(21.9)$ \\
\hline 2 & 4,938 (19.78) & Donor+/recipient- & $4,150(16.62)$ \\
\hline 3 & 3,298 (13.21) & Donor+/recipient+ & $9,680(38.78)$ \\
\hline 4 & $1,839(7.37)$ & Undiagnosed & 2,773 (11.11) \\
\hline 5 & $793(3.18)$ & Delayed graft function & $9,626(38.56)$ \\
\hline 6 or more & $347(1.39)$ & Induction immunosupression & $12,026(48.18)$ \\
\hline \multicolumn{2}{|c|}{ Elixhauser comorbidities } & \multirow{2}{*}{\multicolumn{2}{|c|}{$\begin{array}{l}\text { Discharge immunosupression (ref }=\text { tacrolimus } \\
\text { and MMF) }\end{array}$}} \\
\hline 0 & 4,481 (17.95) & & \\
\hline 1 & $3,294(13.2)$ & Cyclosporine and MMF & $9,009(36.09)$ \\
\hline 2 & $3,453(13.83)$ & Cyclosporine and azathioprine & $3,638(14.57)$ \\
\hline 3 & 3,415 (13.68) & Tacrolimus and MMF & $6,066(24.3)$ \\
\hline 4 & 2,929 (11.73) & Other & $6,250(25.04)$ \\
\hline 5 & 2,411 (9.66) & Steroid Use & 23,339 (93.49) \\
\hline 6 & $1,891(7.58)$ & & \\
\hline 7 & $1,302(5.22)$ & & \\
\hline 8 & $869(3.48)$ & & \\
\hline 9 & $479(1.92)$ & & \\
\hline 10 & $249(1)$ & & \\
\hline 11 or more & $190(0.76)$ & & \\
\hline
\end{tabular}

the complete OPTN model are available upon request.

Lower Medicare payments and shorter LOS were observed in more recent transplant years in a graded relationship. Other factors associated with decreases in Medicare payments and LOS included polycystic kidney disease (PKD) as cause of ESRD and recipient or donor other than white or AfricanAmerican race. In general, the associated with reduced LOS showed statistical significance while the same variables were associated with reduced payments compared to the reference groups but without reaching statistical significance at the 95\% level. One exception was for transplant year of 1997 which also showed significantly reduced Medicare payments.

\section{Charlson and Elixhauser comorbidities}

The top 10 Charlson Comorbidites associated with increased LOS consisted of a mix of OPTN and non-OPTN recorded comordibities. The list included: liver disease (moderate or severe), peptic ulcer disease, congestive heart failure, cerebrovascular disease, diabetes (with and without complications), cancer, rheumatic disease, chronic pulmonary disease and myocardial infarction. Six of the Charlson comorbidities associated with highest increase in Medicare payments were also on the top 10 conditions associated with higher increases in LOS. The other four were: AIDS/HIV, dementia, peripheral vascular disease and mild liver disease (Table III). No Charlson comorbidities were associated with statistically significantly reduced LOS or reduced payments.

Top 10 Elixhauser comorbidities associated with the largest increases in LOS were: lymphoma, other neurological disorders, obesity, peptic ulcer disease excluding bleeding, pulmonary disorders, hypothyroidisms, congestive heart failure, complicated diabetes, alcohol abuse and fluid and electrolyte disorders. Eight of these conditions were also among the top 10 associated with highest increase in Medicare payments. The two other conditions were AIDS/HIV and uncomplicated diabetes (Table III).

Deficiency anemia (-1.5\% [-2.7-0.2\%]), and complicated hypertension $(-1.3[-2.3-0.3])$ were the only factors associated with small and statistically significant reductions in LOS. No Elixhauser comorbidity was associated with statistically significant reductions in payments. 


\section{Sum of Charlson and Elixhauser comorbidities}

Holding all other variables at mean values, each additional Charlson comorbiditiy was associated with a $2.94 \%$ (2.63-3.24) increase in LOS and $\$ 471$ (330-612) additional Medicare payments per patient. Each additional Elixhauser comorbidity carried a 1.71\% (1.5-1.9) increase in LOS and \$277 (202-351) additional Medicare payments.

\section{Donation after cardiac death donor and expanded criteria donor}

Use of a DCD was associated with increases of $14 \%$ in LOS (9.18 to 10.5 days) and 5\% in Medicare payments $(\$ 27,524$ to $\$ 29,018)$. Use of ECD kidneys showed 11 to $15 \%$ increased LOS (9.18 to 10.23 days for donor age $>60$ years and 9.18 to 10.5 days for donor age $>60$ years and hypertension) and 1 to $3 \%$ increase in Medicare payments that was not statistically significant $(\$ 504$ for donor age $>60$ years and $\$ 837$ adding hypertension as donor comorbidity).

\section{Discussion}

Patients considered for transplantation are now older and have more comorbidities than historically [4]. Outcomes are also in general worse with less than standard criteria donor (SCD) kidneys which are used with increasing frequency. However, transplantation of older, sicker patients with less than SCD kidneys has been shown to be more costeffective than remaining on dialysis $[17,18]$. Given these trends, it is important to study the associations between pre-transplant comorbidities and organ quality with transplant procedure LOS and payments.

We confirmed previously reported OPTN factors associated with higher Medicare payments or LOS, such as longer duration of dialysis before transplant, African American race, and use of a DCD [2, 8]. Type I diabetes as cause of ESRD was the leading factor associated with highest LOS and the second one associated with higher Medicare payments. Use of an ECD was previously found not to influence LOS and hospital charges [3], or not strongly influencing Medicare payments [1]. However, other studies indicate that use of an ECD is associated with inadequate payment [1] and longer LOS [2]. We observed that use of a DCD is among the most important factors associated with higher LOS and we also found the use of an ECD to be among the top individual contributors to LOS growth. The associations for ECD and DCD are more likely related to the higher rate of DGF associated with these organs, which will require more dialysis and therefore cost more to the transplant center [19].

Regarding OPTN and commonly considered comorbidities in single center studies, our models

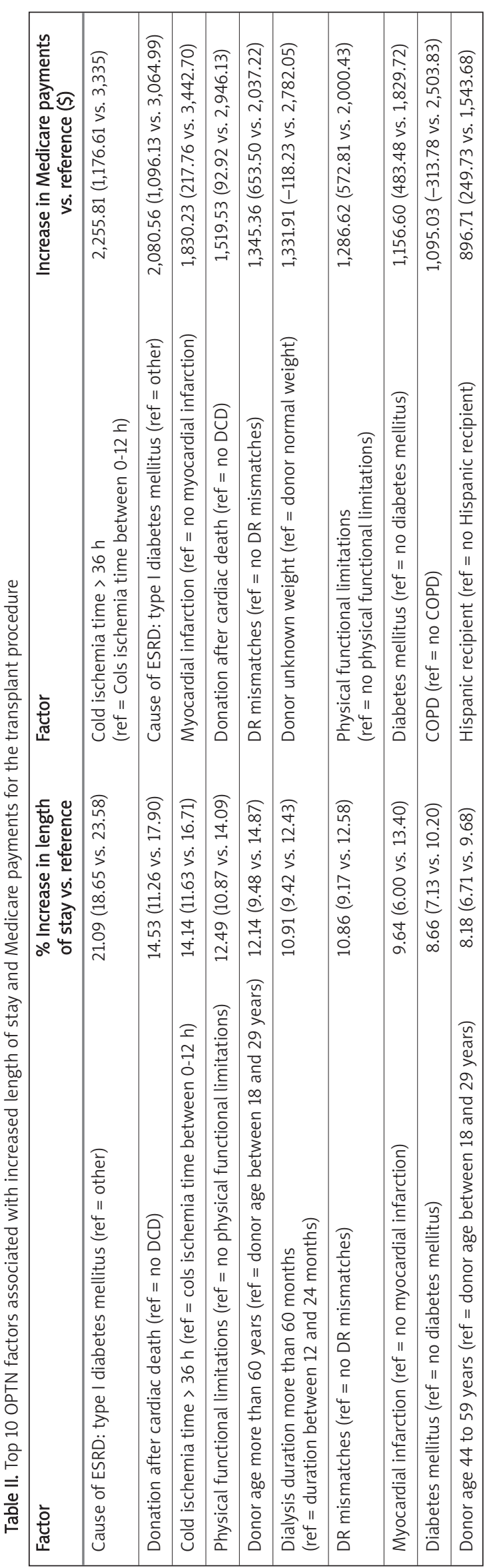




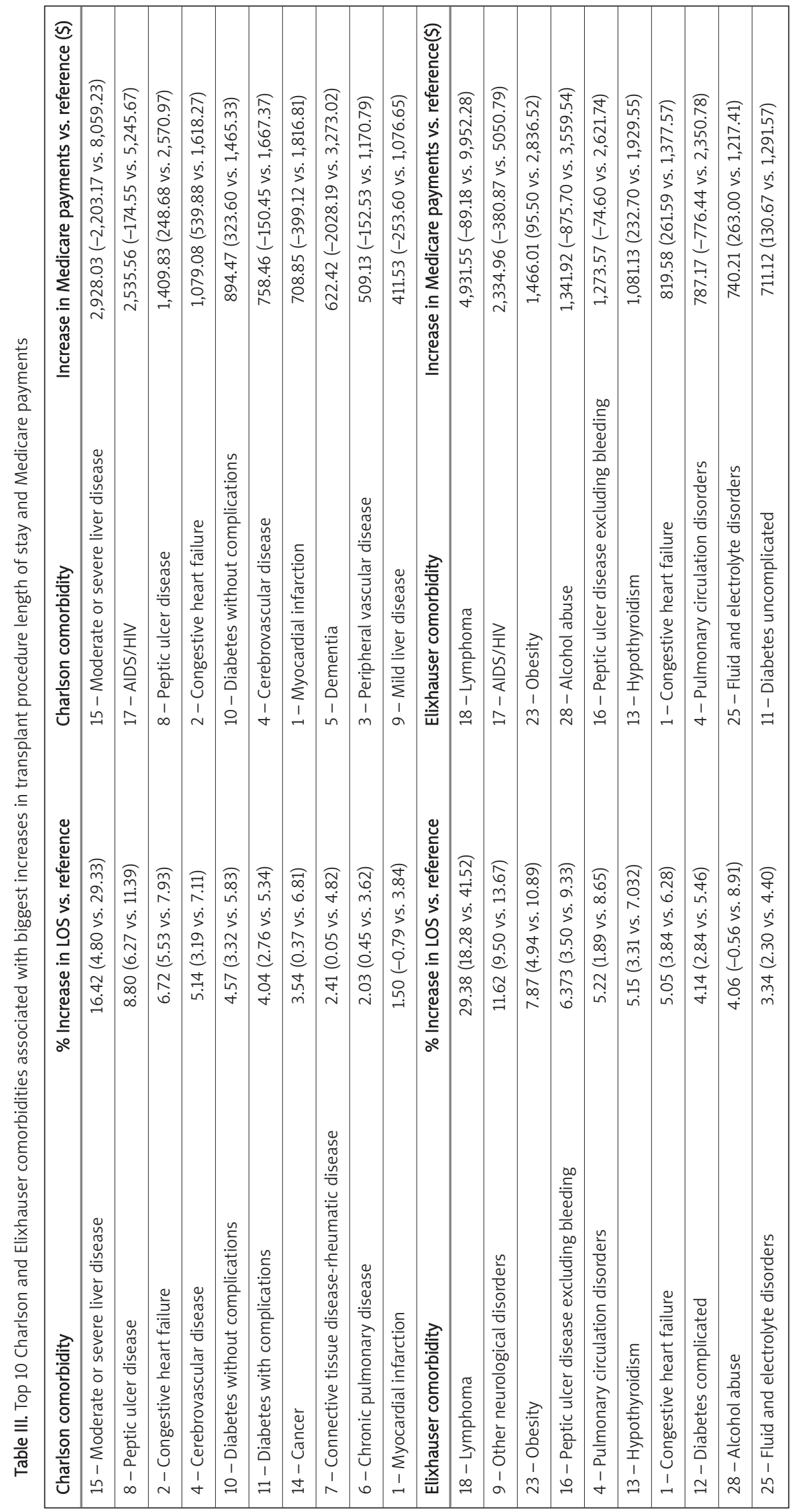


confirmed previous findings. In effect, longer LOS was associated with obesity, COPD and cerebrovascular disease as previously reported in a single center study [8]. These factors were not among those most importantly associated with transplant LOS. Other OPTN reported comorbidities were shown to be associated with higher LOS or Medicare payments either in their OPTN recorded version or in the Charlson or Elixhaser relevant measure (diabetes, myocardial infraction, cerebrovascular disease and obesity). Some non-OPTN reported comorbidities were also among the top contributors to LOS and payments, among them liver disease, cancer, AIDS/HIV, dementia and peptic ulcer disease. Higher costs associated with certain comorbidities are probably reflecting higher rates of early complications leading to longer length of stay or more resource intensive care associated with the comorbid diseases.

Each additional Charlson and Elixhauser comorbidity increased both LOS and Medicare payments and the same was observed for ECD or DCD kidneys, Further analyses should explore if there is a need of increased payments based on comorbid disease or organ quality in order to preserve the financial viability of kidney transplantation to more severe patients or when using ECD or DCD kidneys. Actual hospital cost data, which were not available in this sample would be needed to answer that question,

There are a number of strengths in this research. We add to current knowledge by examining a large number of comorbidities. We also used a multilevel regression method that takes into account potential error correlation between patients in the same centers. Finally, our sample size expands the generalizabiliy of previous work, which relied on single center information.

This study had also some limitations. Selection bias may have occurred because the study sample is restricted to Medicare patients receiving transplants from deceased donors with at least a year on dialysis and the sample may differ for patients who are privately insured. Comorbidity and other model information may have retained some level of measurement error. We expect that the bias would result in conservative estimates.

In conlusion, in this paper we expanded the knowledge of the associations between comorbidities and transplant procedure LOS and Medicare payments beyond known OPTN recorded factors. Further study of associations of pretransplant comorbidities with potential inadequate payments for transplantation warrants consideration. Future work should also examine subtypes of costs, including cost of immunosuppressive regimens. International research should also be pursued. Successful projects aimed at reducing population health risks are known in developing and developed countries [20], and interventions aimed at improving the costeffectiveness of care should also be pursued in groups with important diseases such as chronic kidney disease including transplant recipient. The impact of comborbidities on reimbursement, LOS and transplant finances will depend on local practice, outcomes and reimbursement systems and further studies should be conducted to confirm these associations and design appropriate interventions.

\section{Acknowledgments}

Funding sources: Supported in part by a grants from the National Institute of Diabetes, Digestive, and Kidney, Diseases K25-DK-02916-03, (MAS), PhD, P.I. NIDDK, K08-0730306 (KLL), P30 DK079333 (DCB).

\section{References}

1. Englesbe MJ, Ads Y, Cohn JA, et al. The effects of donor and recipient practices on transplant center finances. Am J Transplant 2008; 8: 586-92.

2. Saidi RF, Elias N, Kawai T, et al. Outcome of kidney transplantation using expanded criteria donors and donation after cardiac death kidneys: realities and costs. Am J Transplant 2007; 7: 2769-74.

3. Sellers MT, Velidedeoglu E, Bloom RD, et al. Expandedcriteria donor kidneys: a single-center clinical and shortterm financial analysis - cause for concern in retransplantation. Transplantation 2004; 78: 1670-5.

4. Weinhandl ED, Snyder JJ, Israni AK, Kasiske BL. Effect of comorbidity adjustment on CMS criteria for kidney transplant center performance. Am J Transplant 2009; 9: 506-16.

5. Englesbe MJ, Dimick JB, Fan Z, Baser O, Birkmeyer JD. Case mix, quality and high-cost kidney transplant patients. Am J Transplant 2009; 9: 1108-14.

6. Abecassis MM. Financial outcomes in transplantation a provider's perspective. Am J Transplant 2006; 6: 1257-63.

7. Axelrod DA, Schnitzler M, Salvalaggio PR, Swindle J, Abecassis MM. The economic impact of the utilization of liver allografts with high donor risk index. Am J Transplant 2007; 7: 990-7.

8. Johnson CP, Kuhn EM, Hariharan S, Hartz AJ, Roza AM, Adams MB. Pre-transplant identification of risk factors that adversely affect length of stay and charges for renal transplantation. Clin Transplant 1999; 13: 168-75.

9. Shwartz M, lezzoni LI, Moskowitz MA, Ash AS, Sawitz E. The importance of comorbidities in explaining differences in patient costs. Med Care 1996; 34: 767-82.

10. Charlson ME, Pompei P, Ales KL, MacKenzie CR. A new method of classifying prognostic comorbidity in longitudinal studies: development and validation. J Chronic Dis 1987; 40: 373-83.

11. Elixhauser A, Steiner C, Harris DR, Coffey RM. Comorbidity measures for use with administrative data. Med Care 1998; 36: 8-27.

12. lezzoni LE. Risk Adjustment for measuring health care outcomes. Chicago, IL.: Health Administration Press 2003. 
13. Quan H, Sundararajan V, Halfon P, et al. Coding algorithms for defining comorbidities in ICD-9-CM and ICD-10 administrative data. Med Care 2005; 43: 1130-9.

14. Luke D. Multilevel Modeling. Thousand Oaks: Sage Publications 2004.

15. Singer JD. Using SAS Proc Mixed to fit multilevel models, hierarchical models, and individual growth models. J Ed Behav Stat 1998; 24: 323-55.

16. Pedan A. Analysis of count data using the SAS system. SUGI 26; April 22-25; Long Beach, CA2001.

17. Jassal SV, Krahn MD, Naglie G, et al. Kidney transplantation in the elderly: a decision analysis. J Am Soc Nephrol 2003; 14: 187-96.

18. Schnitzler MA, Lentine KL, Burroughs TE. The cost effectiveness of deceased organ donation. Transplantation 2005; 80: 1636-7.

19. Buchanan PM, Lentine KL, Burroughs TE, Schnitzler MA, Salvalaggio PR. Association of lower costs of pulsatile machine perfusion in renal transplantation from expanded criteria donors. Am J Transplant 2008; 8: 2391-401.

20. White F, Nanan D. Community health case studies selected from developing and developed countries common principles for moving from evidence to action. Arch Med Sci 2008; 4: 358-63. 